\title{
Effect of surface roughness and substrate material on carbon erosion and deposition in the TEXTOR tokamak
}

\author{
A Kreter ${ }^{1}$, S Brezinsek ${ }^{1}$, T Hirai ${ }^{2}$, A Kirschner ${ }^{1}$, K Krieger ${ }^{3}$, M Mayer ${ }^{3}$, \\ V Philipps ${ }^{1}$, A Pospieszczyk ${ }^{1}$, U Samm ${ }^{1}$, O Schmitz ${ }^{1}$, B Schweer ${ }^{1}$, G Sergienko ${ }^{1}$, \\ K Sugiyama $^{3}$, $T$ Tanabe ${ }^{4}$, Y Ueda ${ }^{5}, P_{\text {Wienhold }}{ }^{1}$ and the TEXTOR team \\ ${ }^{1}$ Institut für Energieforschung - Plasmaphysik, Forschungszentrum Jülich, \\ Association EURATOM-FZJ, Trilateral Euregio Cluster, Germany \\ ${ }^{2}$ Institut für Energieforschung - 2, Forschungszentrum Jülich, Association \\ EURATOM-FZJ, Germany \\ ${ }^{3}$ Max-Planck-Institut für Plasmaphysik, EURATOM Association, Garching, Germany \\ ${ }^{4}$ Interdisciplinary Graduate School of Engineering Science, Kyushu University, \\ Hakozaki 6-10-1, Higashi-ku, Fukuoka 812-8581, Japan \\ ${ }^{5}$ Graduate School of Engineering, Osaka University, Osaka 565-0871, Japan \\ E-mail: a.kreter@fz-juelich.de
}

\begin{abstract}
The technique of ${ }^{13} \mathrm{CH}_{4}$ tracer injection through test limiters was applied to study the influence of surface roughness and substrate material on the local ${ }^{13} \mathrm{C}$ deposition. Spherically shaped graphite limiters with integrated gas injection were prepared with two different grades of surface roughness, $\sim 0.1 \mu \mathrm{m}$ and $\sim 1 \mu \mathrm{m}$. In addition, tungsten limiters with a roughness of $\sim 0.1 \mu \mathrm{m}$ were used to study the substrate material effect. The limiters were exposed to the SOL plasma for two discharge scenarios, Ohmic and neutral beam heated. The ${ }^{13} \mathrm{C}$ deposition efficiency - the ratio of the locally deposited to the injected amount of ${ }^{13} \mathrm{C}$ - and the deposition pattern were evaluated by post-mortem surface analysis. Surface roughness has a pronounced effect and increases the ${ }^{13} \mathrm{C}$ deposition efficiency on graphite with rougher surface by a factor of 3-5 compared to smoother graphite. On tungsten a factor of 2-4 less carbon is deposited than on graphite with similar surface roughness. A systematically higher amount of ${ }^{13} \mathrm{C}$ (by a factor 1.5-2.5) was deposited on limiters exposed to Ohmic compared to neutral beam heated plasmas.
\end{abstract}

PACS numbers: 28.52.Fa, 52.40.Hf, 52.55.Fa, 82.80.Ch, 82.80.Ms

\section{Introduction}

ITER will start operation with a combination of different materials for the plasma facing components (PFCs) including beryllium for the main chamber wall, tungsten for the divertor baffle and carbon fibre re-inforced composite (CFC) for the divertor target, the region with the highest heat loads [1]. For this material mix, the issue of tritium retention has been identified to be mainly associated with codeposition of tritium in layers of re-deposited carbon and beryllium [2]. Where and how fast these layers will grow will primarily define the operational period of ITER until the safety limit of $1000 \mathrm{~g}$ tritium stored in the vessel is reached [1]. Carbon is known for its ability to migrate over long distances in a tokamak and to be deposited far away from its origin. As a consequence, carbon sputtered from the divertor target in ITER can be transported to and re-deposited on the tungsten PFCs, affecting the behaviour of these components and the total amount of tritium co-deposition. Previous investigations on TEXTOR revealed a pronounced difference in erosion and deposition behaviour of carbon for low-Z (graphite) and high-Z (tungsten, molybdenum) substrate materials, with net deposition decreasing for heavier elements. For test limiters of a spherical shape the difference in the local deposition efficiency of injected ${ }^{13} \mathrm{C}$ was of more than one order of magnitude: $4 \%$ for the 
graphite limiter (unpolished, rough) and $0.3 \%$ for the tungsten, which is inherently smoother than graphite [3]. The spherically shaped limiters had a grazing angle of incidence of the magnetic field in the vicinity of the injection aperture. For ${ }^{13} \mathrm{CH}_{4}$ injection experiments using a roof-like geometry with a magnetic field angle of incidence of $20^{\circ}$, the ${ }^{13} \mathrm{C}$ deposition efficiency on polished, smooth plates was $0.17 \%$ for graphite, $0.14 \%$ for molybdenum and $0.11 \%$ for tungsten [4]. A similar effect of the substrate material was found for test mirrors exposed in TCV, showing reduced deposition of background carbon on molybdenum mirrors with respect to silicon mirrors [5], which favours the use of high-Z mirrors in ITER.

Recently, experimental results with spherically shaped limiters exposed in TEXTOR [3] were simulated by the coupled code ERO-SDTrimSP, which, in contrast to the standard version of the ERO code [6], handles more precisely the material mixing effects taking place in the surface layers [7]. The modelling, which assumed flat surfaces, reproduced well the experimental value for the ${ }^{13} \mathrm{C}$ deposition efficiency for tungsten of $0.3 \%$. For graphite the modelling delivered $1.8 \%$, a smaller value than that of $4 \%$ in the experiment. A similar approach to study the dynamics of carbon erosion and deposition on different substrates has been applied in the modelling by the EDDY code $[8,9]$.

Remarkably, the difference in the ${ }^{13} \mathrm{C}$ deposition efficiency for the smooth roof-like limiters [4] was not as pronounced as for the rough spherical limiters [3]. Also the ERO-SDTrimSP modelling struggled to reproduce the large difference in the ${ }^{13} \mathrm{C}$ deposition efficiency for the rough graphite and (inherently smoother) tungsten limiters. These findings triggered additional activities, described in this paper, to investigate the influence of surface roughness on the carbon erosion and deposition behaviour.

The effect of surface roughness on material sputtering has been discussed before, both in experiment and modelling. In particular, sample exposures to ion beams have shown that for rough surfaces the dependence of the sputtering yield on the angle of incidence of beam particles is not as strong as for smooth surfaces [10,11]. Ruzic and co-workers included surface roughness into the TRIM code modelling by means of a statistical description of the fractal geometry $[12,13,14]$. While applicable to atomic-scale roughness only, the model could reproduce the experimental dependence [11] of carbon self-sputtering yields on the angle of incidence for rough surfaces. Küstner et al. used a different approach to numerically simulate material exposure by ion beams, incorporating a real sample topography measured by scanning tunnelling microscopy into the TRIM model $[15,16]$. In addition to the well reproduced influence of the angle of incidence on sputtering for rough surfaces, they found that a significant fraction of sputtered atoms can instantly be re-deposited on a rough surface due to geometry effects. This leads effectively to a decrease of the sputtering yield.

Previous studies in tokamaks revealed a non-uniform erosion-deposition behaviour of impurities due to rough target surfaces. For instance, after exposure of a graphite substrate pre-coated with a boron film in the erosion-dominated zone in TEXTOR, $\sim 10 \mu \mathrm{m}$ large "island"-like structures of remaining boron were found in the recessed areas of the graphite surface [17,18]. Similarly, a non-uniform distribution of deposited material was found on graphite tiles in the erosion-dominated outer divertor of ASDEX-Upgrade [18]. Surface topography was found to influence the erosion characteristics of both carbon and tungsten in JET [19] and ASDEX-Upgrade [20]. Nevertheless, the common opinion is that the influence of surface roughness on erosion and deposition in tokamaks, in contrast to ion beam experiments, is not as significant. In support of this, particle trajectories in presence of a magnetic field are complicated and result in a broad distribution of angles of incidence of impinging particles. Therefore, the effect of surface roughness on erosion and deposition in tokamaks is, if at all, considered as an increase of the effective area due to local surface wrinkles. Modelling, both interpretative for the present day machines and predictive for the next generation devices including ITER, mostly does not include the effect of surface roughness.

The aim of the experiments presented here was to investigate quantitatively both the effects of surface roughness and of the substrate material on the erosion and deposition behaviour of carbon for tokamak plasma conditions. This paper provides the evidence that, indeed, surface roughness influences the erosion/deposition processes at PFCs, and that, for a reactor relevant range of roughness, this influence is at least as strong as the effect of substrate materials. The experiments should provide a basis for benchmarking while incorporating the surface roughness effect in the modelling tools, e.g. the ERO code, which should improve the quality of their predictions for ITER. 
The paper is organized as follows: Section 2 describes the experiments at TEXTOR; Post-mortem surface analysis is subject of section 3; In section 4 the results are summarized, possible mechanisms responsible for the effects of surface roughness and substrate material are discussed.

\section{Limiter preparation and exposure}

Isotropic fine-grain graphite EK98 and bulk polycrystalline tungsten were utilized as low-Z and high$\mathrm{Z}$ limiter materials, respectively. Limiters with different surface topography, which was precharacterized by a stylus profiler, were used for the experiments: (i) unpolished, rough graphite limiters with an average surface roughness of $R a \sim 1 \mu \mathrm{m}$, (ii) polished, smooth graphite limiters with $R a \sim 0.1 \mu \mathrm{m}$ and (iii) roughened tungsten limiters with $R a \sim 0.1 \mu \mathrm{m}$. The limiters had a spherical shape with a size of $120 \mathrm{~mm}$ in toroidal direction, $80 \mathrm{~mm}$ in poloidal direction and a curvature radius of $70 \mathrm{~mm}$.

The limiters were exposed to the scrape-off layer (SOL) plasma of TEXTOR, a medium size tokamak (major radius $R_{0}=1.75 \mathrm{~m}$, minor radius $a=0.46 \mathrm{~m}$ ) with circular plasma cross-section. Results for the tungsten and rough graphite limiters used under conditions with neutral beam injection (NBI) heating were previously published in [3]. The referred paper describes in detail the application of the TEXTOR limiter lock system for the sample exposure as well as the geometry of the spherically shaped test limiters. The new experiments include exposures of a smooth graphite limiter for the same plasma conditions and the limiter position (the top of the limiter at a minor radius of $r=0.48 \mathrm{~m}$, $20 \mathrm{~mm}$ outside the last closed flux surface) as well as another set of tungsten, rough and smooth graphite limiters, all three exposed to Ohmic plasmas at $r=0.47 \mathrm{~m}$. The methane gas injection aperture was situated $15 \mathrm{~mm}$ away from the top of the limiter in toroidal direction, so that the radial position of the aperture was $1.7 \mathrm{~mm}$ behind the limiter top. This geometry of the experiment assures that the upper part of the limiters is located in the erosion dominated zone of the SOL. The vicinity of the injection aperture turned into a net deposition zone due to the methane injection. All limiters were pre-heated to $\approx 400^{\circ} \mathrm{C}$. During the plasma discharges, the surface temperature excursions were not larger than $50^{\circ} \mathrm{C}$.

Each of the limiters was exposed to 6-10 TEXTOR discharges in deuterium, all with ${ }^{13} \mathrm{CH}_{4}$ injection. The pulse duration was 5-6 s with flattop values of the toroidal magnetic field of $2.25 \mathrm{~T}$ and of the plasma current of $350 \mathrm{kA}$. NBI heated discharges had a central line-averaged electron density of $3.5 \cdot 10^{19} \mathrm{~m}^{-3}$ and a total heating power of $1.5 \mathrm{MW}$, Ohmic pulses of $2.5 \cdot 10^{19} \mathrm{~m}^{-3}$ and $0.3 \mathrm{MW}$, respectively. Refer to figure 3 in [3] for time traces of a typical NBI heated discharge scenario. Typical profiles of the edge electron density and temperature measured by the He-beam diagnostic for both heating scenarios are shown in figure 1. For these SOL plasma parameters, the relevant characteristic lengths such as ion gyroradii and the surface sheath thickness of a few Debye lengths are much larger than the typical surface roughness.

The technique of ${ }^{13} \mathrm{C}$-marked methane gas injection was applied to provide a well know local carbon source and to distinguish in the post-mortem analysis between injected ${ }^{13} \mathrm{C}$ and co-deposited ${ }^{12} \mathrm{C}$ from the background plasma. The methane gas was injected through an aperture in the limiter surface with a diameter of $2 \mathrm{~mm}$ for all but the tungsten limiter in the NBI heated case $(1 \mathrm{~mm})$. Nevertheless, ${ }^{13} \mathrm{CH}_{4}$ injection rates of $\sim 10^{19} \mathrm{~s}^{-1}$ were similar in all six experiments. No influence on global plasma parameters including carbon concentration in the plasma was observed. However, as was shown in [4], the injection can have a noticeable influence on local plasma parameters. No data is available on the plasma parameters in the vicinity of the injection, but a local density increase with a simultaneous cooling of plasma can be expected during the gas puff. The change of local plasma parameters, however, should be similar for otherwise equal discharge scenarios and similar injection rates. Therefore, the influence of local plasma parameters can be excluded when comparing the results for different limiters exposed to the same discharge scenario.

Visual observation of the limiters during their exposures showed a continuous and constant growth of the deposition during the methane injection. In all exposure series, no abnormal events took place which would change the erosion/deposition behaviour significantly. The amount of ${ }^{12} \mathrm{C}$ in the background plasma, which was identified to influence the ${ }^{13} \mathrm{C}$ deposition efficiency [4], was similar in all discharges with the same heating scenario. 


\section{Carbon deposition on limiters}

Figure 2 shows photographs of the limiters after their exposures. Deposited carbon formed hard amorphous layers on the limiter surfaces with a maximum thickness of 1-2 $\mu \mathrm{m}$ and a deuterium content of $10 \%-20 \%$, determined by surface analysis techniques as described below. The elongated shape of the ${ }^{13} \mathrm{C}$ deposits near the injection aperture is attributed to the preferred direction of the local carbon transport defined by the direction of the SOL plasma flow combined with the ExB drift, whereby $\mathrm{E}$ is the radial electric field (cf. [3]). According to the stylus profiler measurements, the plasma exposure did not significantly alter the surface roughness of the limiters in both erosion and deposition dominated zones.

Comparing the limiters with the same exposure scenario (NBI heated or Ohmic), a pronounced difference in the ${ }^{13} \mathrm{C}$ deposition pattern on the limiter surfaces can be seen for the smooth versus rough graphite limiters. The area covered by the carbon layer near the injection aperture is about four times larger for the rough than for the smooth limiters. The ${ }^{13} \mathrm{C}$ deposition pattern on the smooth graphite limiters has an area about twice as large as on the tungsten limiters. The ${ }^{13} \mathrm{C}$ films deposited on both tungsten limiters have well defined margins, whereas they are more blurred on the smooth graphite limiters. The ${ }^{13} \mathrm{C}$ films are separated from the ${ }^{12} \mathrm{C}$ deposition from the background plasma by a net erosion zone for the tungsten as well as for the smooth graphite limiters. In case of the rough graphite limiters the ${ }^{13} \mathrm{C}$ deposition expanded so strongly that it overlaps with the background ${ }^{12} \mathrm{C}$ deposition.

A combination of surface analysis techniques was applied to determine the integral amounts of deposited elements and their depth and surface distributions. The surfaces of all limiters were investigated by interference fringe analysis, a method to relate colours produced by deposited semitransparent films to deposition thicknesses. For a known layer density, it allows the determination of the total carbon $\left({ }^{12} \mathrm{C}+{ }^{13} \mathrm{C}\right)$ amount in the deposited film. Depth profiles of different elements and isotopes, including the isotopic ratio of ${ }^{13} \mathrm{C} /{ }^{12} \mathrm{C}$, were measured for graphite limiters by secondary ion mass spectrometry (SIMS). The tungsten limiters were too massive to apply the SIMS technique. Absolute areal densities of ${ }^{13} \mathrm{C},{ }^{12} \mathrm{C}$ and $\mathrm{D}$ were measured by nuclear reaction analysis (NRA) with a $2.5 \mathrm{MeV}{ }^{3} \mathrm{He}$-beam. The protons from the ${ }^{12} \mathrm{C}\left({ }^{3} \mathrm{He}, \mathrm{p}\right){ }^{14} \mathrm{~N},{ }^{13} \mathrm{C}\left({ }^{3} \mathrm{He}, \mathrm{p}\right){ }^{15} \mathrm{~N}$ and $\mathrm{D}\left({ }^{3} \mathrm{He}, \mathrm{p}\right){ }^{4} \mathrm{He}$ reactions were detected with a large angle particle counter equipped with a stopper foil for filtering of backscattered ${ }^{3} \mathrm{He}$ ions. The smooth graphite and tungsten limiters were analysed by NRA twodimensionally for the entire deposition zone near the injection aperture with a lateral resolution of $\sim 2 \mathrm{~mm}$. Because of its wide deposition pattern, the rough graphite limiter exposed to NBI heated plasma was analysed by NRA at a few selected positions only. No NRA data are available for the rough graphite limiter exposed to the Ohmic plasma. The ${ }^{13} \mathrm{C}$ amount can be gathered either from NRA or from a combination of SIMS and interference fringe analysis. The cross-check of these methods gave an agreement within $30 \%$.

The two-dimensional distribution of ${ }^{13} \mathrm{C}$ deposition gathered by NRA and normalized by the amount of injected molecules is shown in figure 3. It has its maximum close to the injection aperture and is more extended for the smooth graphite than for the tungsten limiters with a similar roughness. Figure 4 shows the normalized ${ }^{13} \mathrm{C}$ deposition along the axis of widest extension of the deposited films. Here, the ${ }^{13} \mathrm{C}$ amounts were provided by NRA with exception of the rough graphite limiter from Ohmic discharges. For the latter SIMS in combination with interference fringe analysis was used. The colour pattern of the deposited film close to the injection aperture is unclear, leading to lack of measurement points in this region. Again, the main difference in ${ }^{13} \mathrm{C}$ deposition is its significantly wider distribution on the rough graphite compared to the smooth limiters. Deposition on tungsten shows the lowest extension.

Based on the data obtained by surface analysis, the total amounts of the local ${ }^{13} \mathrm{C}$ deposition and the corresponding deposition efficiency were determined for each limiter. Table 1 summarizes the data, showing significantly higher deposition efficiencies for rough versus smooth graphite limiters. Also, the deposition efficiency on low- $Z$ graphite is larger than on high- $Z$ tungsten limiters of similar roughness. A systematically higher ${ }^{13} \mathrm{C}$ fraction is deposited in Ohmic than in NBI heated discharges. Injected ${ }^{13} \mathrm{C}$, which was not locally deposited, was presumably transported by the plasma towards other obstacles at the vessel wall, as it was observed in [4]. 
Table 1. Amounts of injected and deposited ${ }^{13} \mathrm{C}$ and their quotient, deposition efficiency, for six exposed limiters.

\begin{tabular}{|l|c|c|c|c|c|c|}
\hline Scenario & \multicolumn{3}{|c|}{ NBI heated plasma } & \multicolumn{3}{c|}{ Ohmic plasma } \\
\hline Limiter & $\begin{array}{c}\text { Rough } \\
\text { graphite }\end{array}$ & $\begin{array}{c}\text { Smooth } \\
\text { graphite }\end{array}$ & Tungsten & $\begin{array}{c}\text { Rough } \\
\text { graphite }\end{array}$ & $\begin{array}{c}\text { Smooth } \\
\text { graphite }\end{array}$ & Tungsten \\
\hline${ }^{13} \mathrm{CH}_{4}$ injected & $5.5 \cdot 10^{20}$ & $1.7 \cdot 10^{20}$ & $5.7 \cdot 10^{20}$ & $2.2 \cdot 10^{20}$ & $2.8 \cdot 10^{20}$ & $2.7 \cdot 10^{20}$ \\
\hline${ }^{13} \mathrm{C}_{\text {deposited }}$ & $2.0 \cdot 10^{19}$ & $2.3 \cdot 10^{18}$ & $1.5 \cdot 10^{18}$ & $2.0 \cdot 10^{19}$ & $4.7 \cdot 10^{18}$ & $2.3 \cdot 10^{18}$ \\
\hline $\begin{array}{l}{ }^{13} \mathrm{C} \text { deposition } \\
\text { efficiency }\end{array}$ & $4 \%$ & $1.3 \%$ & $0.3 \%$ & $9 \%$ & $1.7 \%$ & $0.8 \%$ \\
\hline
\end{tabular}

\section{Summary and discussion}

The effects of surface roughness and substrate material on the erosion-deposition behaviour of carbon have been studied by means of ${ }^{13} \mathrm{CH}_{4}$ tracer injection through tungsten and graphite limiters. The latter had two different grades of surface roughness. The tungsten limiters had surface roughnesses similar to smooth graphite.

The local ${ }^{13} \mathrm{C}$ deposition is more efficient in Ohmic than in NBI heated discharges, despite similar plasma parameters in the SOL measured by the He-beam diagnostic. One possible explanation for this can be a different plasma positioning during both types of discharges, which can result in different edge plasma parameters at the position of the test limiters (bottom of the poloidal cross-section), whilst the He-beam, placed at the low field side, still measures similar plasma parameters. Note that the core plasma parameters in the NBI and Ohmic pulses were significantly different (i.e. higher plasma pressure in the NBI case), which could have influenced the plasma positioning system.

The ${ }^{13} \mathrm{C}$ deposition efficiency on tungsten is lower by a factor of 2-4 than on graphite with similar roughness for both applied exposure scenarios, NBI heated and Ohmic. Surface layers with a thickness of $\sim 10 \mathrm{~nm}$ protect the substrate from the impinging particles with an energy of $\sim 100 \mathrm{eV}$, typical for the TEXTOR SOL [21]. For thicker layers, the substrate material should not influence the erosion and deposition processes at the top surface. The maximum of the layer thickness near the injection aperture reaches this value within one TEXTOR pulse. However, the differences in the evolvement of deposition on tungsten and graphite are still observable after several pulses. Likely, the margin of the deposited layer, which is always subjected to the influence of the substrate material, defines the further evolvement of the deposition pattern.

For the spherical limiter geometry with grazing angles of incidence of the magnetic field used in this study, the effect of the substrate material is stronger than for the previously investigated roof-like geometry with an angle of incidence of $20^{\circ}$. For the latter, the difference in the ${ }^{13} \mathrm{C}$ deposition efficiency for graphite and tungsten was of the order of $50 \%$ [4]. The influence of the geometry can presumably be attributed to the key factors of the substrate effect discussed in $[3,7]$ : (i) higher kinetic reflection coefficients of carbon on tungsten than on graphite and (ii) the enhanced physical sputtering of carbon deposited on tungsten. Both of these factors are strongly geometry dependent.

The effect of substrate material would lead to less extended deposition dominated zones on high-Z than on low-Z PFCs in a long-pulse device due to a different balance between local deposition and erosion. Thus, tungsten surfaces are expected to be less affected by carbon deposition in ITER. This favourable effect is better pronounced for ITER-relevant grazing angles of incidence of the magnetic field.

The graphite limiters polished to $R a \sim 0.1 \mu \mathrm{m}$ collect significantly less carbon than the unpolished limiters with $R a \sim 1 \mu \mathrm{m}$. The difference in the ${ }^{13} \mathrm{C}$ deposition efficiency by a factor of 3-5 is mainly due to a wider expansion of the deposition pattern in case of the rough limiters. Obviously, the balance between local gross deposition and erosion is positive for a larger area on the rough limiters.

One possible explanation for higher net deposition on the rough surface is a local shadowing of surface recessions and pores from the incoming hydrogen ions responsible for erosion. This effect was observed for $0.3 \mathrm{~mm}$ deep marker holes in a special tile of the main limiter in TEXTOR [22,23] as well as for a test limiter with a local shadow created by a $3 \mathrm{~mm}$ thick plate [24]. In both cases, deposition took place in the recessed areas, whereas the adjacent surface was a net erosion zone. The origin of the deposition in local shadows is the difference in the Larmor radii for particles contributing 
to deposition or erosion. Hydrocarbon molecules and radicals in the TEXTOR SOL have gyroradii of several millimetres, which makes the recessed areas accessible for them. In contrast, a large fraction of deuterium ions with gyroradii of $<1 \mathrm{~mm}$ are scraped off by the local barrier and cannot reach the shadowed regions and erode carbon from there. However, in our case surface pits have a much smaller characteristic depth than Larmor radii of all ion species in plasma. Therefore, surface roughness shall not change the relative numbers of incident particles and switch the local erosion-deposition balance by this mechanism.

A more probable explanation of the surface roughness effect is the enhanced prompt re-deposition of physically eroded carbon on rough surfaces. This mechanism was proposed in $[15,16]$ to explain the differences in sputtering yields as a function of the angle of incidence of particles for rough and smooth surfaces. Figure 5 illustrates this mechanism. While for a flat surface all eroded atoms escape it in the first instance, atoms sputtered from a rough surface can scrape the local wrinkles and thus be promptly re-deposited. The probability for the eroded particles to be "trapped" this way increases with surface roughness. Therefore, this mechanism can change the local balance of deposition and erosion, thus increasing the amount of locally stored ${ }^{13} \mathrm{C}$ for the rough limiters with respect to the smooth.

We conclude that surface roughness plays an important role for erosion and deposition of PFCs in present-day tokamaks. To account for the effect of surface roughness, it is not sufficient only to increase the effective area due to local surface wrinkles, keeping the extension of the deposition pattern constant. Instead, the local balance between deposition and erosion on rough surfaces turns in favour of deposition, thus increasing the extension of the deposition dominated zone.

Most impurity transport codes do not consider surface roughness. Instead, the simulations attribute impacts of surface roughness to other effects. In consequence, it increases uncertainties of their predictions for the material migration and tritium retention in ITER. Therefore, it is necessary to include surface roughness in the codes. The results of this paper are suitable to serve for benchmarking of such modelling studies.

In a long-pulse device like ITER, the erosion-deposition behaviour of the surfaces will strongly depend on mechanisms of material erosion in long-term exposures. Higher deposition in pores and recessions and enhanced erosion at hilltops and ridges, as was observed in [19,20], may finally result in a smoothing of the initially rough technical surfaces. This effect of long pulse and steady-state operation is difficult to investigate in today's machines, but may have a large relevance in future experiments.

\section{References}

[1] Progress in the ITER Physics Basis 2007 Nucl. Fusion 47 S1

[2] Kirschner A et al 2007 J. Nucl. Mater. 363-365 91

[3] Kreter A et al 2006 Plasma Phys. Control. Fusion 481401

[4] Kreter A et al 2007 J. Nucl. Mater. 363-365 179

[5] De Temmerman G et al 2008 Fusion Eng. Des. 8330

[6] Kirschner A et al 2000 Nucl. Fusion 40989

[7] Droste S et al 2008 Plasma Phys. Control. Fusion 50015006

[8] Ohya K et al 2004 Physica Scripta 111138

[9] Ohya K et al 2005 J. Nucl. Mater. 337-339 882

[10] Haasz A A, Davis J W and Wu C H 1989 J. Nucl. Mater. 162-164 915

[11] Roth J, Bohdansky J and Ottenberger W 1989 J. Nucl. Mater. 165193

[12] Ruzic D N and Chiu H K 1989 J. Nucl. Mater. 162-164 904

[13] Ruzic D N 1990 Nucl. Instr. Meth. B 47118

[14] Brooks J N and Ruzic D N 1990 J. Nucl. Mater. 176-177 278

[15] Küstner M, Eckstein W, Dose V and Roth J 1998 Nucl. Inst. Meth. B 145320

[16] Küstner M, Eckstein W, Hechtl E and Roth J 1999 J. Nucl. Mater. 26522

[17] Wienhold et al 1999 J. Nucl. Mater. 266-269 986

[18] Hildebrandt D et al 1999 Physica Scripta T81 25

[19] Mayer M et al 2007 J. Nucl. Mater. 363-365 101

[20] Mayer M et al 2007 Physica Scripta T128 106

[21] Maier H, Schmid K and Eckstein W 2005 J. Nucl. Mater. 337-339 480

[22] Mayer M et al 2003 J. Nucl. Mater. 313-316 377 
[23] Kreter A et al 2007 Physica Scripta T128 35

[24] Wienhold et al 2001 Physica Scripta T94 141 

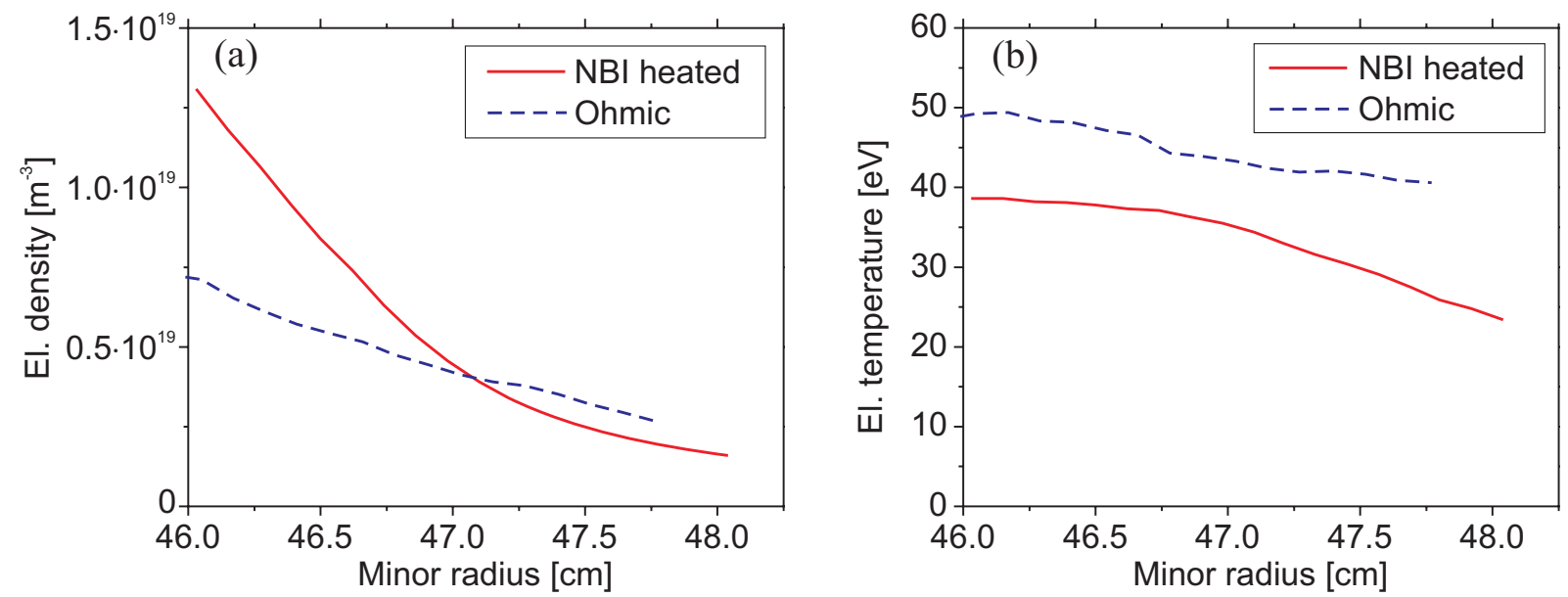

Figure 1. Radial electron (a) density and (b) temperature profiles in the SOL for discharges with limiter exposure. The LCFS is at $46 \mathrm{~cm}$. 

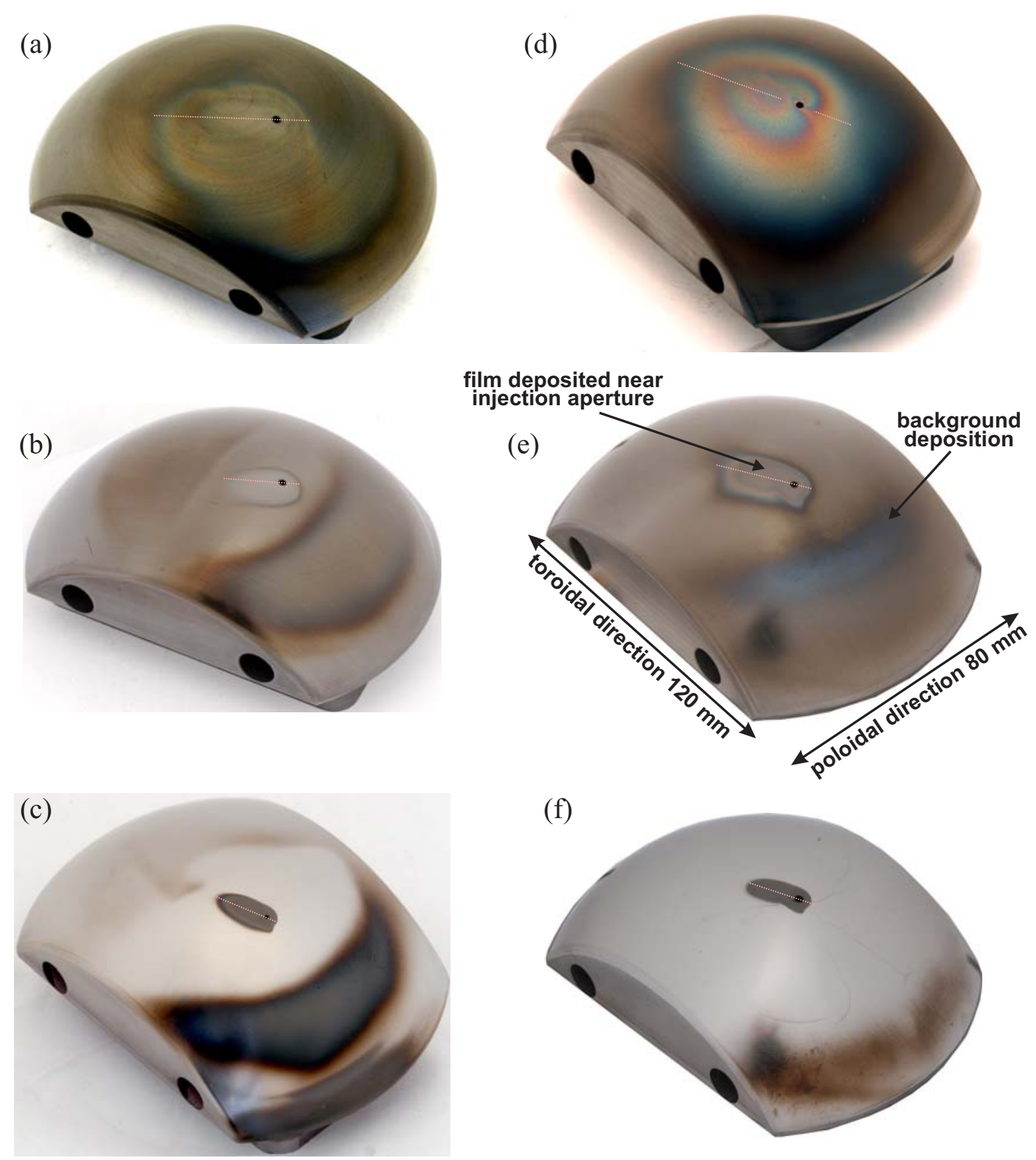

Figure 2. Photographs of limiters exposed in (a-c) NBI heated and (d-f) Ohmic discharges. (a), (d) rough graphite limiters, (b), (e) smooth graphite limiters and (c), (f) tungsten limiters. Broken lines across the injection apertures indicate the positions of the ${ }^{13} \mathrm{C}$ line scans in figure 4 . 
(a) Normalized ${ }^{13} \mathrm{C}$ areal density $\left[\left({ }^{13} \mathrm{C}_{\text {dep }} / \mathrm{cm}^{2}\right){ }^{13} \mathrm{C}_{\text {ini }}\right]$

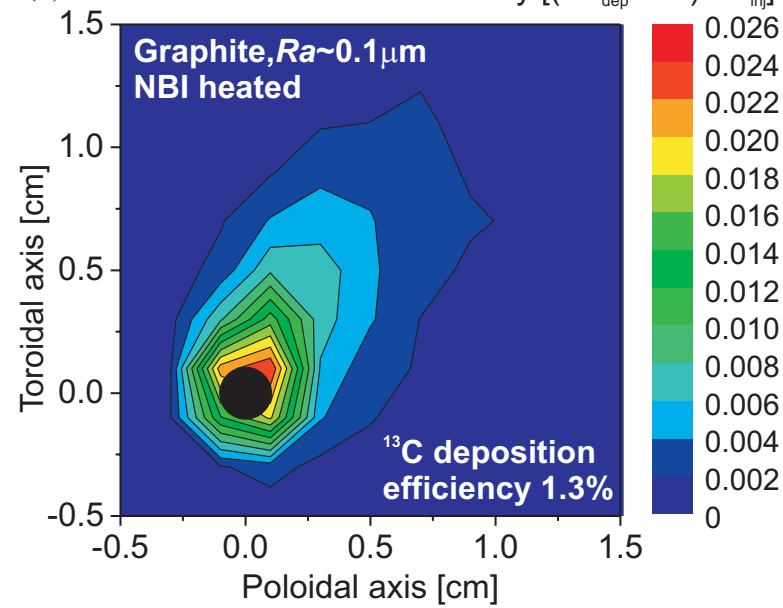

(b) Normalized ${ }^{13} \mathrm{C}$ areal density $\left.\left[\left({ }^{13} \mathrm{C}_{\text {dep }} / \mathrm{cm}^{2}\right)\right]^{13} \mathrm{C}_{\text {ini }}\right]$

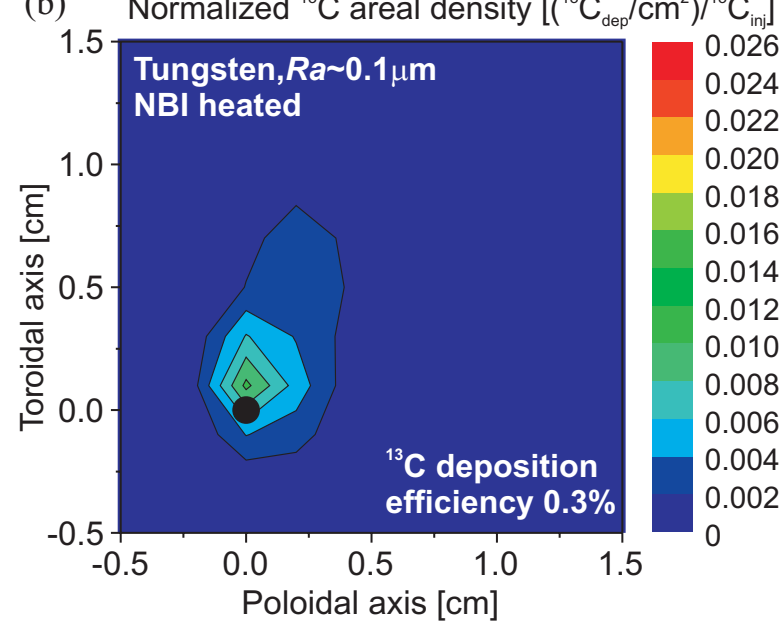

(c) Normalized ${ }^{13} \mathrm{C}$ areal density $\left.\left[\left({ }^{13} \mathrm{C}_{\text {dep }} / \mathrm{cm}^{2}\right)\right)^{13} \mathrm{C}_{\text {ini }}\right]$

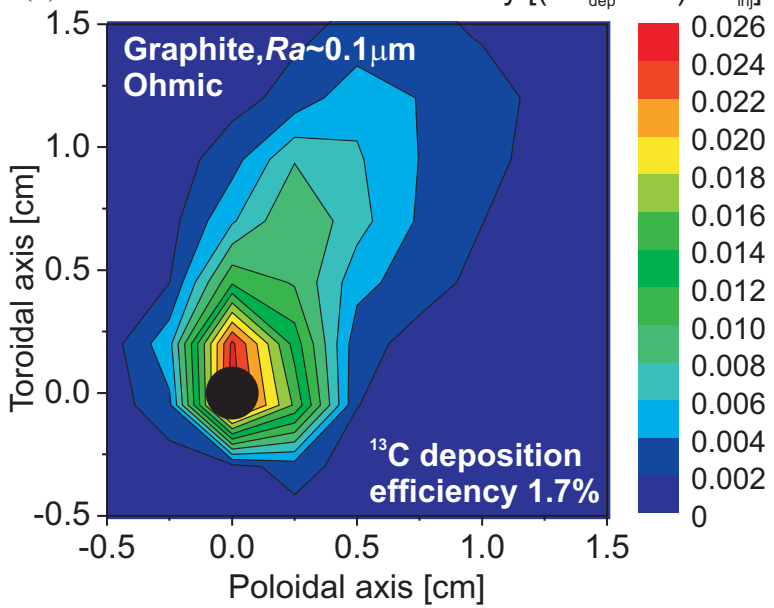

(d) Normalized ${ }^{13} \mathrm{C}$ areal density $\left[\left({ }^{13} \mathrm{C}_{\text {dep }} / \mathrm{cm}^{2}\right) /{ }^{13} \mathrm{C}_{\text {inin }}\right]$

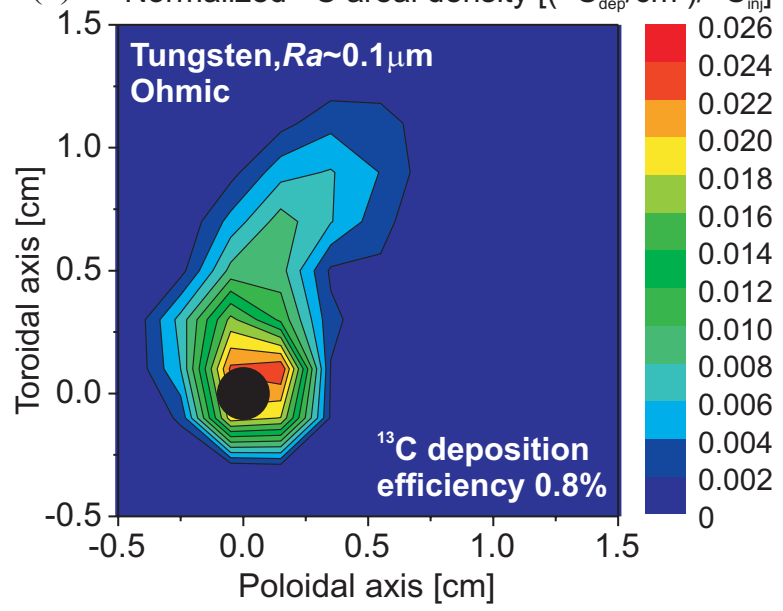

Figure 3. Two-dimensional surface distribution of ${ }^{13} \mathrm{C}$ deposited near the injection aperture for (a), (c) smooth graphite and (b), (d) tungsten limiters exposed in (a), (b) NBI heated and (c), (d) Ohmic discharges. The deposited ${ }^{13} \mathrm{C}$ amount is normalized by the amount of injected ${ }^{13} \mathrm{CH}_{4}$. Black circles represent the injection apertures. 

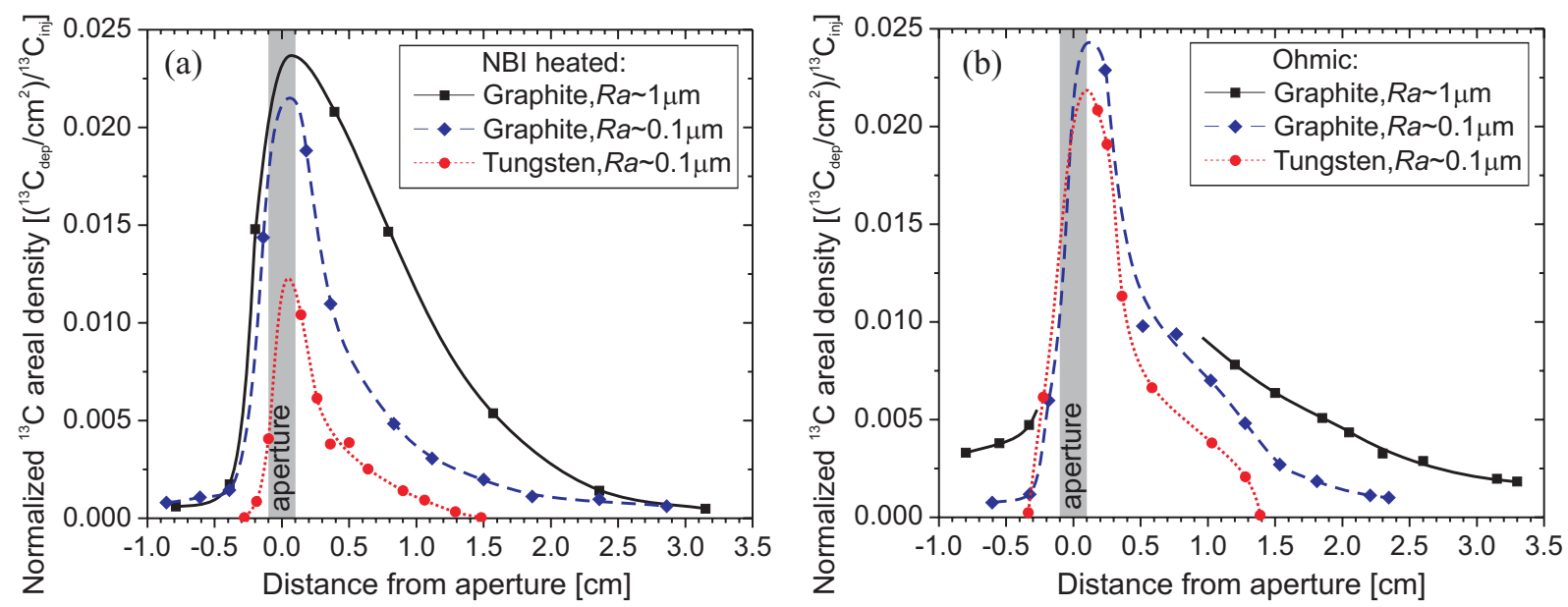

Figure 4. Amounts of ${ }^{13} \mathrm{C}$ deposited near the injection apertures along lines indicated in figure 2 for limiters exposed in (a) NBI heated and (b) Ohmic discharges. Symbols indicate the measurement points; curves are to guide the eye. The deposited ${ }^{13} \mathrm{C}$ amount is normalized by the amount of injected ${ }^{13} \mathrm{CH}_{4}$. 
(a)

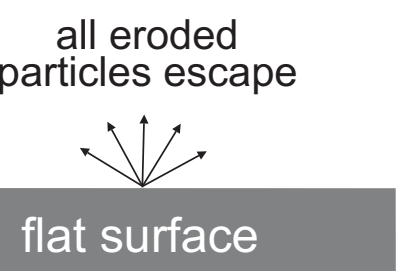

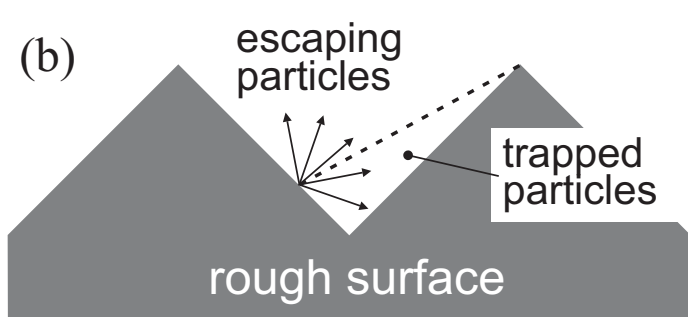

Figure 5. Illustration of the enhanced prompt re-deposition on rough surfaces. (a) all eroded atoms can escape from a flat surface; (b) a fraction of atoms eroded from a certain position with trajectories below the broken line will hit the rough surface and be promptly re-deposited, thus decreasing the effective sputtering yield. 